\title{
A comparison of complication rates in a single unit between parenteral and enteral nutrition following upper gastrointestinal resection surgery
}

\author{
S. F. Stevenson, R. Fulton, M. Panarelli and R. F. McKee \\ Department of Nutrition, Glasgow Royal Infirmary, 84-106 Castle Street, Glasgow, G4 OSF, UK
}

Background: There is conflicting data on the recommended route of nutrition post-operatively following an upper gastrointestinal resection $^{(1)}$. Traditionally enteral nutrition is associated with a lower complication rate ${ }^{(2)}$. We sought to compare the infective and mechanical complication rate between the parenteral and enteral feeding in a single unit which manages both practices.

Methods: We went through theatre lists dating back to August 2007, the formation of the upper GI surgery department in Glasgow Royal selecting patients who had an upper GI resection and required post-operative nutrition. Data were collected using patients' notes from case notes, dietician records and electronic patient records. Data were subsequently analysed using the mini-tab statistical program.

\begin{tabular}{lcrc}
\hline Outcome & Parenteral & Enteral & $P$ value \\
\hline Overall Mortality (\%) & 22.73 & 36.00 & 0.117 \\
Mortality at 1 year (\%) & 18.18 & 16.00 & 0.758 \\
\% Weight Change (mean) & 97.89 & 95.13 & 0.148 \\
Length of Stay (mean) & 23.65 & 21.10 & 0.403 \\
Duration of Support (mean) & 11.91 & 11.59 & 0.832 \\
Infective Complications (\%) & & & \\
Chest & 50.00 & 40.00 & 0.283 \\
Urine & 16.67 & 4.00 & $\mathbf{0 . 0 1 8}$ \\
Collection & 16.67 & 6.00 & 0.081 \\
Stool & 6.06 & 4.00 & 0.620 \\
Line/Feeding Tube & 0.00 & 2.00 & 0.312 \\
Wound & 14.06 & 14.00 & 0.992 \\
Mechanical Complications (\%) & & & \\
Blockage & 1.64 & 14.29 & $\mathbf{0 . 0 1 6}$ \\
Dislodged & 3.33 & 6.12 & 0.489 \\
Removed due to pyrexia & 24.59 & 0.00 & $\mathbf{0 . 0 0 0}$ \\
Pneumothorax & 1.60 & 4.10 & 0.454 \\
Arterial Puncture & 3.20 & 0.00 & 0.145 \\
Feed leak & 1.60 & 4.10 & 0.556 \\
Diarrhoea & 2.00 & 4.00 & 0.379 \\
Constipation & 0.00 & 11.10 & $\mathbf{0 . 0 3 4}$ \\
Perforation & 0.00 & 4.10 & 0.144 \\
\hline
\end{tabular}

Results: We identified 116 patients from the period of Aug 2007 to Oct 2010. 66 patients received parenteral nutrition (TPN) and 50 enteral (EN) nutrition; feeding jejunostomy (46) or nasojejunal feed (4). There were no statistically significant differences in overall mortality to date, percentage of body weight changed from admission, length of stay or duration support in days. See table. The mean length of stay however for EN patients was 2 days less than PN (21.10 compared to 23.65 days). Overall, $68.18 \%$ of patients on PN had an infective complication of any kind compared to $52.00 \%$ of patients on EN, P value 0.075 . The only significant specific difference was a higher incidence in urinary tract infections in PN patients. In terms of mechanical complications, the PN group had a higher incidence of line removal due to unexplained pyrexia although this often proved to be due to others causes other than a line infection. EN patients had a higher rate of constipation and tube blockage. In addition, although not statistically significant ( $\mathrm{P}$ value 0.224$)$, there were 2 incidences of perforations associated with EN, one directly related to volvulus formation around the jejunostomy tube and subsequent small bowel perforation requiring a laparotomy.

Conclusions: Parenteral nutrition was associated with a slight although not significant increased infection rate and length of stay. There was a statistically significant higher incidence of urinary tract infections; and lines removal due to unexplained pyrexia. The greater mean length of stay of 2 days in the PN group may not be of statistical significance but may have clinical significance in terms of increased hospital days and may reflect the greater overall infection rate in the PN group. Enteral nutrition was more likely to lead to constipation and tube blockages. In addition, in this series led to a very severe complication relating to the tube itself. Although, this suggests a greater degree of interrupted nutrition in the EN group, this does not appear to be significant on review of the outcomes measured.

1. Pacelli F, Bossala M, Papa V, Sgadari A \& Doglietto G (2002) Lancet 359, 1697-1998.

2. Mazaki T \& Ebisawa K (2008) J Gastrointest Surg 12, 739-755. 